\title{
Assembly Process Bill Of Material Construction Technology For Spacecraft Assembly And Integration Based On Proengineer
}

\author{
WAN Bi-le ${ }^{1}, H E$ Wen-xing ${ }^{*}$, CHEN Chang-yu ${ }^{1}$ \\ *1. Beijing Institute of Spacecraft Environment Engineering, Beijing 100094, China
}

Keywords: Digital Assembly; BOM; Pro/E;PDM.

\begin{abstract}
Spacecraft is a complex electromechanical thermocouple system, which composed of several subsystems and thousands of products. The spacecraft assembly process is to assemble the products in the main structure of the spacecraft, and completes the relevant detection and testing. The spacecraft development unit can be divided into overall design, subsystem design and manufacturing integration department. This paper introduces the PBOM automatic construction principle and workflow based on Pro/E. Finally, this paper describes the improved efficiency and reliability of the software.
\end{abstract}

\section{Introduction}

Spacecraft is a complex electromechanical thermocouple system, which composed of several subsystems and thousands of products. The spacecraft assembly process is to assemble the products in the main structure of the spacecraft, and completes the relevant detection and testing. The spacecraft development unit can be divided into overall design, subsystem design and manufacturing integration department.

In recent years, based on Model Based Definition (MBD), domestic and foreign universities and enterprises have carried out digital product design and manufacturing technology research. Min Liu et al. studied the transformation between EBOM (Engineering Bill of Material) and maintainability BOM [1]; Chen Zhi et al. used BOM to carry out research on multi-product joint inventory management technology, which involved BOM construction technology [2]; Jang Hyun Lee et al. proposed a suitable BOM construction technology for shipbuilding, which can display the product structure and information [3]; Gonen Singer et al. proposed a BOM conversion technology from plan to production for small batch of complex products, which can add additional information in the BOM [4]; Tsai Chi Kuo built a low-carbon products BOM in PDM system through the standardized data collection forms and search engine system [5]; C. MartinezOlvera studied the influence of BOM on the process [6]; Wang Weiguo and others studied storage model of manufacturing BOM [7]; Liu Xiaobing and others studied BOM multi-view conversion technology based on dummy BOM [8].

Aerospace development enterprises generally complete the spacecraft three-dimensional design by the overall design and manufacturing integration department, and then the spacecraft three-dimensional models are sent to the manufacturing integration department through the interface with the overall design and manufacturing integration department. The manufacturing integration department constructs the manufacturing integration $\mathrm{PBOM}$ based on the spacecraft three-dimensional models (i.e. EBOM), and then carries out digital process design and manufacturing integration work. However, for the above spacecraft assembly PBOM construction technology, there are still the following questions:

(1) The spacecraft system is complex, according to product usage, it can be divided into instruments, direct parts, thermal control components, fasteners, cables, pipelines and so on, and the assembly relationship among various types of products is complex.

(2) The overall design uses a professional top-down division method. EBOM is respectively organized and designed in accordance with multiple professions such as mechanical, electrical, thermal control, etc. Assembly integration departments need to interpret the information in accordance with the product assembly relationship, that is, the EBOM is visualized to construct the desired PBOM.

(3) The fastener information in EBOM is generally expressed in the parameter table or database without the physical modeling of fasteners or other standard parts. Assembly and integration departments view the output table of parameter or database to obtain relevant information, and they are instantiated in the PDM system in order to facilitate the application of process design and assembly implementation.

(4) In general, the traditional PBOM construction method is manually created by the craftsman in the PDM system, which is inefficient and unreliable. 
In the analysis of PBOM construction process and the spacecraft assembly integration, it is not hard to find:

(1) Although there is a wide range of spacecraft products (including a total of 24 categories: instruments, pipe fittings, pipe fittings, insulation pads, insulation film, grounding components, fasteners, electrical connectors, straight parts, cables, thermistors, heating circuit, Heating, multi-layer, thermocouple, external heat flow, equipment accessories, piping, ventilation systems, thermal control foam and flame retardant cloth, cabin structure, hose, interior decoration and pipe fittings). In order of simplifying the expression of product assembly relationship ,the 24 categories of products can be simplified to seven categories of objects, consisting of instrument, cable, piping, heating circuit, direct parts, deck and thermal control multi-layer (known as main product object), and the others are affiliated products (known as Affiliated Product Object) .

(2) The PBOM construction of Spacecraft assembly integration is essentially analyzing the product assembly relationship displayed in the form of BOM, which will be instantiated in the form of parameters or database. The process is the visualization of BOM.

(3) Assembly integration PBOM can be automatically analyzed and visual processing in the CAD software, and then imported into PDM, which improves work efficiency and quality.

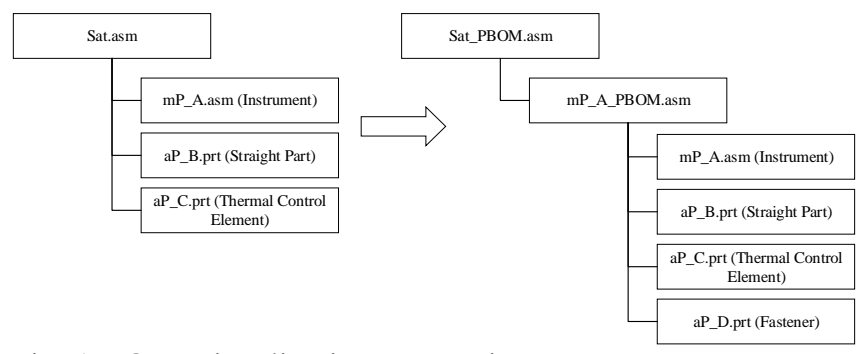

Fig. 1 BOM Visualization Processing

According to the overall design division of the spacecraft, the whole satellite model is composed of three types of professional models: the whole mechanical structure models including satellite deck structure, instrument, straight parts and others; the electrical product models including cable, electrical connector and other affiliated product, etc.; the thermal control product models including thermistor, thermocouple, heating circuit and heating film, thermal control multi-layer etc. The spacecraft assembly integration department needs to assemble the models as an integral satellite model in accordance with the whole satellite coordinate system after receiving the design models, as shown in Figure 1. It is gradually constructed out of each corresponding PBOM node to the main object in Pro/E, and the whole satellite PBOM node, Sat_PBOM.asm.

For the assembly integration department, five categories of objects including cable, piping, heating circuit, deck, thermal control multi-layer and their affiliated objects in EBOM have fulfilled the task requirements. In this paper, instruments, straight parts and their affiliated fasteners are taken as examples to illustrate the PBOM visualization process by using the Pro/E software.

\section{Fundamental Principles}

Set all node elements of the whole satellite PBOM as the set $U$, and set $u_{i}$ as a node element of PBOM, then there is: $U=\left\{u_{i}\right\}$

Determining the process of $u_{i}$ is to inherit, find or create the product component objects that meet certain conditions, such as mP_A.asm, aP_B.prt, aP_C.prt, ap_D.prt in Figure 1 and other components or parts.

In addition to fasteners and other standard parts of the product which need to be instantiated and assembled with the corresponding master products, the other product components have a solid model and establish with the whole satellite coordinate system. The determination of such product components needs to determine whether the object is the required one in accordance with certain criteria, the criteria for this paper are as follows:

(1) Product type matching principle, that is, each node element $u_{i}$ has 1 component of master product objects, and other product objects are affiliated product object type, such as mP_A.asm for the instrument of master product objects, aP_B.prt, aP_C.prt for the straight parts and thermal control elements of affiliated product objects.

(2) Assembly constraint principle, that is, based on the matching principle of product types, distinguish whether there is assembly constraints (such as face matching) between the master product mP_A.asm and aP_B.prt, if so, determine aP_B.prt as a subsidiary object of mP_A.asm, and B.prt as a component of A_PBOM.asm.

(3) Geometric relations principle, that is, based on the product type matching principle, calculate the normal distance between the master product object mP_A.asm and aP_C.prt, if the distance is the shortest in the product master objects and other affiliated product objects, and the normal distance between aP_C.Prt and other product objects is larger than the value, then determine aP_C.prt as an affiliated product object of mP_A.asm.

Respectively, set $a_{i} 、 b_{i}, c_{i}$ as the product objects to meet the above criteria, and

$$
\begin{aligned}
& A=\left\{a_{i}\right\} \\
& B=\left\{b_{i}\right\} \\
& C=\left\{c_{i}\right\}
\end{aligned}
$$


According to PBOM construction guidelines, there is

$$
U=(A \cap B) \cup(A \cap C)
$$

Simplified as

$$
U=A \cap(B \cup C)
$$

Let ${ }^{d_{i}}$ be a standard object such as fasteners for instantiation, and

$$
D=\left\{d_{i}\right\}
$$

To determine the $d_{i}$, weneed to obtain product components,such as fasteners, including the number, specifications and standards, through the parameter table or database and then create such product objects in PBOM, including the creation of the corresponding product node (such as aP_D.prt), and assemble it to the corresponding position based on determining its position relative to the whole satellite coordinate system.

Thus we can identify all the constituent elements of the entire satellite PBOM:

$$
U=(A \cap(B \cup C)) \cup D
$$

\section{PBOM Visualization Construction Process}

For spacecraft assembly integration, $\mathrm{PBOM}$ visualization process is based on the BOM reconstruction to build PBOM without changing the assembly relationship of EBOM products, and instantiates fastener product objects in the form of product parameters, thus enabling the visualization of assembly integration information.

In addition to fasteners and other standard products, the visualization of other products should follow the product type matching, assembly constraints, geometric relations and other three principles, the visualization of these products do not need to be instantiated, but need to complete the product $\mathrm{BOM}$ reconstruction and model relocation. Fasteners and other standard products need to read the product assembly with fasteners standards, specifications and other parameters of information to build the corresponding fastener model and the model can be simplified model, and then mounting fastener model in the holes corresponding products.

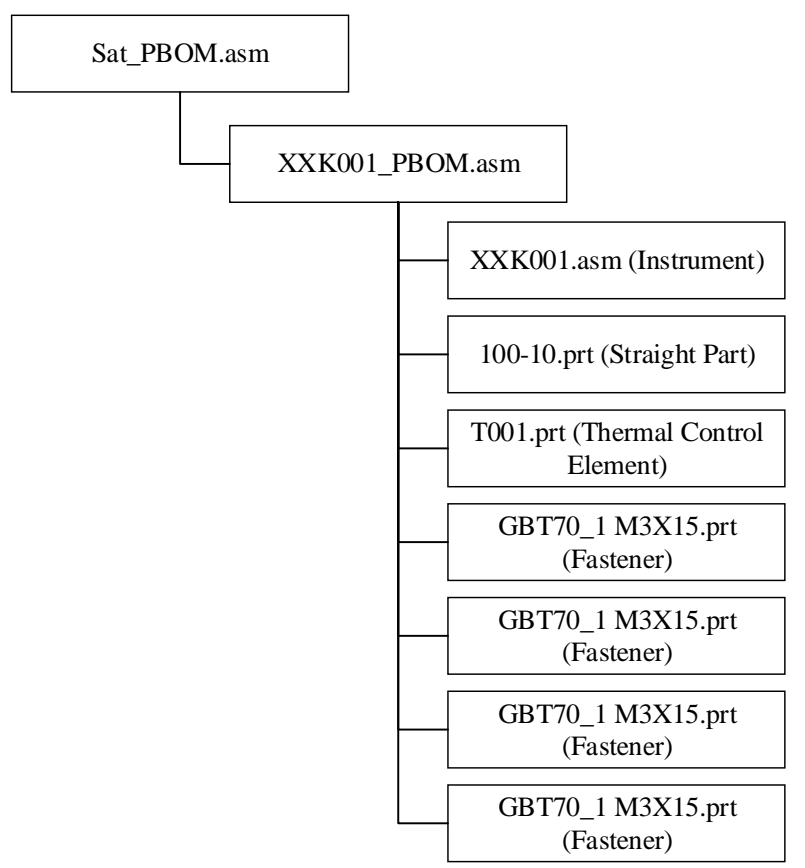

Fig. 2 Example of Satellite Assembly Visualization BOM

Satellite assembly visualization PBOM construction process is shown in Figure 3.

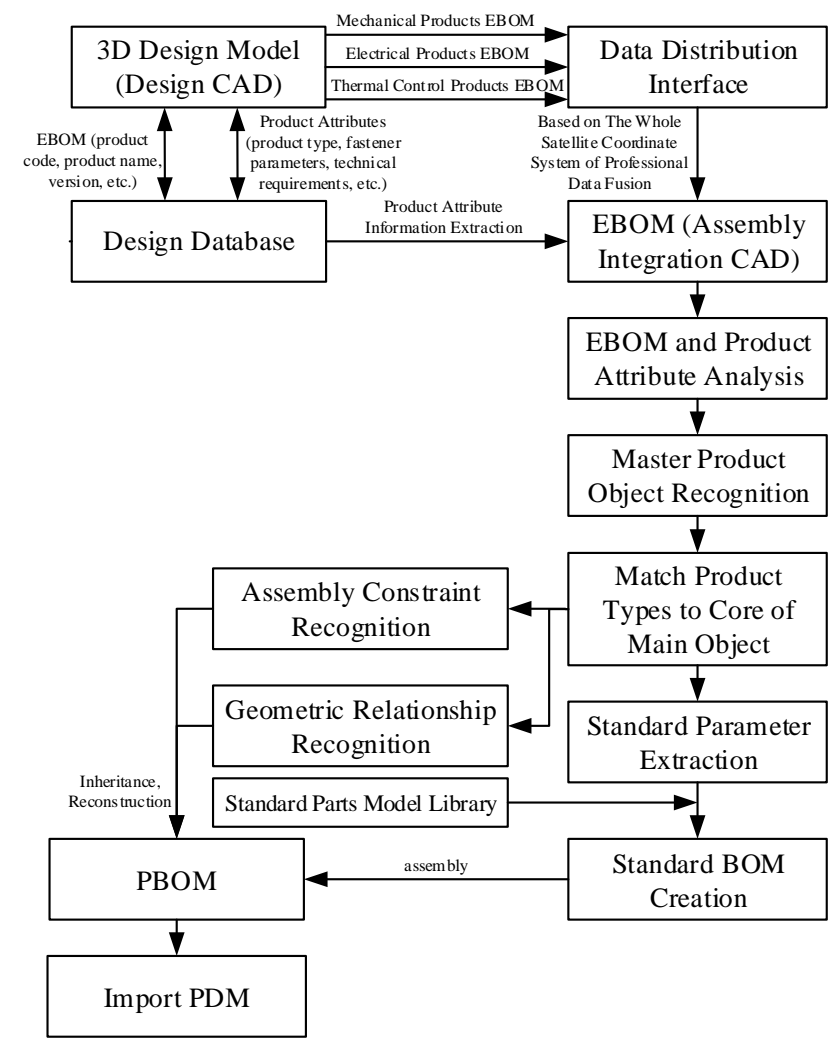

Fig. 3 Satellite Assembly Visualization BOM Process 


\section{System Application}

In this paper, Pro/E and ProToolkit are used to establish a set of PBOM visualization construction system for satellite and other spacecraft assembly, which is applied in the practical model development. The system obtains the controlled 3D model and product attribute information from the design data management system of the overall design department (Avidm4.0 system used in this paper), based on EBOM and its related product attributes, creates a visual PBOM that meets the requirements of the assembly integration department through Inheritance, reconstruction and new, etc., and automatically and import PBOM into the PDM system through interfaces with PDM (Teamcenter for this paper). The system not only achieves the automatic transmission of 37 commonly used satellite assembly integration information (more than $30 \%$ compared to the traditional model), but also increases the efficiency from design data to assembly integration department more than 1 times (from 20 people down to 8 People days).

\section{Conclusion}

This paper presents a visualization PBOM processing method for the integration of spacecraft assembly through variety of assembly information generated in the three-dimensional design process of the design departments, realizes the automatic construction and application of the visualization PBOM of satellite assembly integration, succeeds in transmitting to the PDM System, and greatly improves the efficiency and quality of data transmission. The method has good processing power for mechanical products and related thermal control products. However, it is difficult to deal with the problem of products with complicated assembly relationships and many branches such as cables and pipelines, which need to be focused on in the further.

\section{Acknowledgements}

This research is supported by Natural Science Foundation of China (No. 51405025), and Beijing Spacecraft Smart Assembly and Equipment Engineering Research Center.

\section{References}

[1] LIU M, LAI J, SHEN W M. A method for transformation of engineering bill of materials to maintenance bill of materials.[J]. Robotics and Computer Integrated Manufacturing: An International Journal of Manufacturing and Product and Process Development,2014,30(4).

[2] CHEN Z, REN C, SHAN M Y. Multi-Product of JMI under BOM.[J]. International journal of advanced pervasive and ubiquitous computing,2014,6(4).

[3] JANG H L, SEUNG H K, KYUNGHO L. Integration of evolutional BOMs for design of ship outfitting equipment.[J]. Computer-Aided Design,2012,44(3).

[4]GONEN S, MAYA G, YUVAL C. From product documentation to a 'method prototype' and standard times: a new technique for complex manual assembly.[J]. International journal of production research,2014,52(1/2).

[5]TSAI C K. The construction of a collaborative framework in support of low carbon product design.[J]. Robotics and Computer Integrated Manufacturing: An International Journal of Manufacturing and Product and Process Development,2013,29(4).

[6]C.Martinez-Olvera. An entropy-based approach for assessing a product's BOM blocking effect on a manufacturing process flow.[J]. International journal of production research,2012,50(2/4).

[7] Wang Weiguo, Yan Guangrong, Lei Yi. Novel storage model of manufacturing BOM [J]. Computer Integrated Manufacturing Systems, 2000,16 (3): 558-562.

[8] Liu Xiaobing, Huang Xuewen, Ma Yue, etc. Research on XBOM for Product Whole Life Cycle [J] .Computer Integrated Manufacturing System, 2002,8 (12): 983-987. 